\title{
Kaskadennutzung von Lignocellulose: LX-Verfahren trifft auf B. coagulans
}

\author{
LINDA SCHROEDTER ${ }^{1}$, FRIEDRICH STREFFER ${ }^{2}$, KATRIN STREFFER ${ }^{2}$, PETER UNGER ${ }^{1}$, \\ JOACHIM VENUS ${ }^{1}$ \\ ${ }^{1}$ LEIBNIZ-INSTITUT FÜR AGRARTECHNIK UND BIOÖKONOMIE (ATB), \\ ABT. BIOVERFAHRENSTECHNIK, POTSDAM \\ ${ }^{2}$ LXP GROUP GMBH, TELTOW
}

\section{Investigating alternatives for petrobased substrates, lignocellulose is an interesting yet complex feedstock that offers various possibilities for the design of new and sustainable chemical routes. The novel energy-saving LX-pretreatment was combined with thermophilic Bacillus coagulans. By this, corn straw was used in an innovative cas- cade obtaining biogas, lignin as well as polymerisable L-(+)-lactic acid of over 99 percents optical purity.}

DOI: $10.1007 / \mathrm{s} 12268-020-1499-7$

(C) Die Autoren 2020

Chemische Grundbausteine unseres alltäglichen Bedarfs werden nach wie vor meist auf Basis von Erdöl erzeugt. Doch moderne Biotechnologie strebt danach, unsere Wirtschaftsweise zu revolutionieren: An Stelle von fossilen Energieträgern sollen nachwachsende Rohstoffe, Rest- und Abfallstoffe die Grundlage für die Entwicklung vielfältiger neuer Produktwege bilden [1]. Ein besonders interessanter Rohstoff ist in diesem Zusammenhang die Lignocellulose.

Lignocellulose besteht aus den drei chemischen Strukturen Cellulose, Hemicellulose und Lignin. Cellulose und Hemicellulose enthalten vorrangig Zuckermoleküle, wobei erstere homogen aus Ketten der Glucose und letztere aus Pentosen, wie bspw. der Xylose, besteht. Einmal aus ihren Verbindungen in Lösung gebracht, können diese unter Einsatz von geeigneten Mikroorganismen zu wertvollen Produkten fermentiert werden. Lignin ist dagegen ein sehr komplexes, heterogenes Polymer, dessen vorrangig aromatische Verbindungen die Entwicklung biobasierter Produkte ermöglichen [2]. Erfolgreiche Trennung und Aufbereitung der einzelnen Bestandteile der Lignocellulose sind somit von großem Interesse für die Forschung.

In unserem Verbundprojekt PoMiZu („Alternative Zucker zur Gewinnung von polymerisierbarer Milchsäure“) wurde ein neuartiger Aufschluss von Lignocellulose (LX-Verfahren) mit einer biotechnologischen Umwandlung der Mischzucker zu polymerisierbarer Milchsäure kombiniert. Milchsäure (LA) kommt in der Natur in der Form von zwei stereochemischen Isotopen $\mathrm{L}-(+)^{+}$und D-(-) vor. Liegen diese Moleküle in hoher optischer Reinheit vor, ist mittels Polymerisierung die Produktion des modernen Kunststoffs PLA möglich, der thermisch kompostierbar ist und eine Alternative zu erdölbasierten Kunststoffen bietet.

\section{Vom Stroh zur polymerisierbaren Milchsäure}

Die Landwirtschaft ist einer der Wirtschaftsbereiche in denen jedes Jahr Lignocellulose in großen Mengen anfällt, bspw. in der Form von Stroh. Neben Einstreu in Tierställen und Humusrückbildung kann Stroh energetisch über Verbrennung oder Biogasgewinnung genutzt werden. Im Projekt PoMiZu wurde der Gärrest einer Biogasanlage, die mit Energiemais betrieben wird, verwendet. Das Maisstroh verbleibt zum Gutteil als faserigeres Material im Gärrest. Dieser Rohstoff wurde zunächst von der LXP Group GmbH zur Gewinnung von Lignin mit dem neuartigen LX-Verfahren aufgeschlossen. Die Lignocellulose ist berüchtigt für ihre Widerstandsfähigkeit und wird in der Regel unter Aufwendung von hoher Temperatur und/oder hohem Druck bearbeitet. Neben integriertem Recycling der Arbeitsstoffe liegt der Vorteil des LX-Verfahrens darin, dass besonders milde Prozessbedingungen bei Temperaturen unter $80{ }^{\circ} \mathrm{C}$ und atmosphärischem Druck herrschen. Die Biomasse wird mit Phosphorsäure versetzt, es folgt eine Fällung und Lignin wird mithilfe einer Fest-/Flüssigtrennung über die Suspension abgetrennt. Der zurückgebliebene Feststoff unterläuft mehrere Waschschritte, die der Rückgewinnung

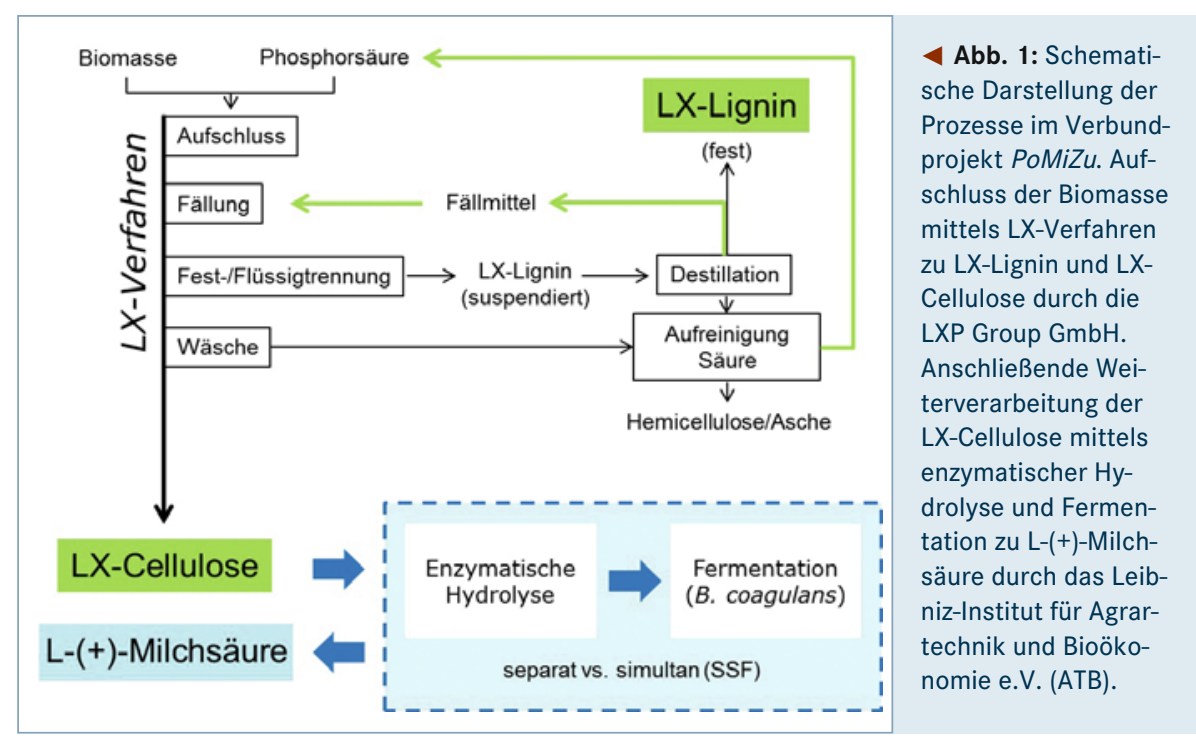


der Phosphorsäure dienen, und kann anschließend enzymatisch hydrolysiert werden, um Monosaccharide für die Fermentation bereitzustellen (Abb. 1). Der Feststoff wird als LX-Cellulose bezeichnet, da im Vergleich zum Ausgangsmaterial sein Anteil an Cellulose erhöht und derjenige der Hemicellulose reduziert ist.

Übliche Aufschlussverfahren von Lignocellulose setzen aufgrund des hohen Energieeinsatzes Inhibitoren wie Furfural und Hydroymethylfurfural (HMF) frei, die das Wachstum der Bakterien be- oder sogar verhindern können. Zudem können viele LAProduzenten die Pentosen der Lignocellulose nicht oder nur ineffizient verwerten [3]. So ist für die Arbeit mit Lignocellulose entscheidend, einen wirkungsvollen Mikroorganismus für die Fermentation zu identifizieren.

\section{Multitalent Bacillus coagulans}

Stämme der Spezies Bacillus coagulans sind in der Lage, sowohl Glucose als auch Pentosen $\mathrm{zu}$ verwerten und dies mitunter sogar zeitgleich. Es entstehen selten Nebenprodukte und erhebliche Konzentrationen an Inhibitorstoffen werden toleriert [4]. Ferner wurde gezeigt, dass B. coagulans cellolytische Enzyme produziert [5]. B. coagulans hat zudem nur simple Nährstoffanforderungen und liefert bereits mit der Zugabe von Hefeextrakt beste Ergebnisse. Doch auch mit weniger kostenintensiven Nährstoffen wie Bäckerhefe konnte B. coagulans in Lignocellulose-Hydrolysat kultiviert werden [6].
B. coagulans gehört $\mathrm{zu}$ den thermophilen Organismen und wächst daher optimal bei erhöhten Temperaturen von $52{ }^{\circ} \mathrm{C}$. Dies reduziert nicht nur das Risiko der Kontamination durch andere LA-Bildner, die in der Regel mesophile Temperaturen von $37{ }^{\circ} \mathrm{C}$ bevorzugen, sondern ermöglicht auch die simultane enzymatische Hydrolyse und Fermentation (simultaneous saccharification \& fermentation, SSF).

Im Projekt PoMiZu sollte geprüft werden, ob eine SSF von LX-Cellulose mit B. coagulans und der Enzymlösung Cellic ${ }^{\circledR}$ CTec2 (Novozymes) möglich ist. Hierzu wurde experimentell geprüft, welches Isolat der Spezies B. coagulans die beste Leistung bei $\mathrm{pH} 5$ erbringt, da dieser Wert dem Optimum der Enzyme entspricht. Isolate wurden im Doppelversuch unter Verwendung des EloFermdual-Systems (Biotronix) in einem Arbeitsvolumen von 0,25 Liter mit automatischer Basezugabe (20\% NaOH) getestet. Als Substrat diente der filtrierte Überstand einer enzymatischen Hydrolyse von LX-Cellulose (Maisgärrest). Der pH-Wert wurde über die Laufzeit der Fermentation bei pH 5,0-5,1 gehalten. Konzentrationen der LA und Zucker wurden mittels high pressure liquid chromatography (HPLC) gemessen. Das beste Ergebnis lieferte Isolat A162 mit 98,8 Prozent maximaler Ausbeute und einer Produktivität von $3,2 \mathrm{~g} \cdot \mathrm{L}^{-1} \cdot \mathrm{h}^{-1}$. Isolat A166 erreicht eine Produktivität von $2,6 \mathrm{~g} \cdot \mathrm{L}^{-1} \cdot \mathrm{h}^{-1}$ bei einer Ausbeute von 84,9 Prozent (Ausbeute auf Basis der Monosaccharide). Beide Stäm- me erreichten die maximale Ausbeute bereits nach nur acht Stunden Fermentation. Dabei ist hervorzuheben, dass neben der Glucose parallel Xylose abgebaut wurde. Zusätzlich weist Isolat A166 für diesen Zeitraum eine geringe Verwertung der Cellobiose auf, dem Disaccharid der Cellulose (Abb. 2). Neben guter Performance in der Fermentation ist jedoch auch das Endprodukt entscheidend. Für Isolat A166 lag L-LA mit einer optischen Reinheit von > 99 Prozent vor, während 98,5 Prozent für Isolat A162 gemessen wurden. Für weitere Versuche wurde sich daher für B. coagulans A166 (DMSZ ID 14-300) entschieden.

\section{Separater und simultaner Prozess im Vergleich}

Der separate und der simultane Prozess wurden mit einem Arbeitsvolumen von einem Liter unter Verwendung eines BIOSTAT ${ }^{\circledR}$ Laborfermenters (Sartorius) durchgeführt, bei automatischer Zufuhr von Base $(20 \%$ $\mathrm{NaOH})$. Die Hydrolyse des separaten Prozesses fand beim Optimum der Enzyme statt $\left(50{ }^{\circ} \mathrm{C}, \mathrm{pH} 5\right)$ und die Fermentation bei optimalen Bedingungen für B. coagulans A166 $\left(52{ }^{\circ} \mathrm{C}, \mathrm{pH}\right.$ 6). Im simultanen Prozess wurde das Optimum der Enzyme gewählt. Im separaten Versuch lagen nach 24 Stunden enzymatischer Hydrolyse 27,1 g · $\mathrm{L}^{-1}$ Monosaccharide vor, die innerhalb von weiteren 23 Stunden $\mathrm{zu} 23,7 \mathrm{~g} \cdot \mathrm{L}^{-1} \mathrm{LA}$ mit einer optischen Reinheit von 99,3 Prozent L-(+)-LA umgewandelt wurden. Im simultanen Versuch wurden
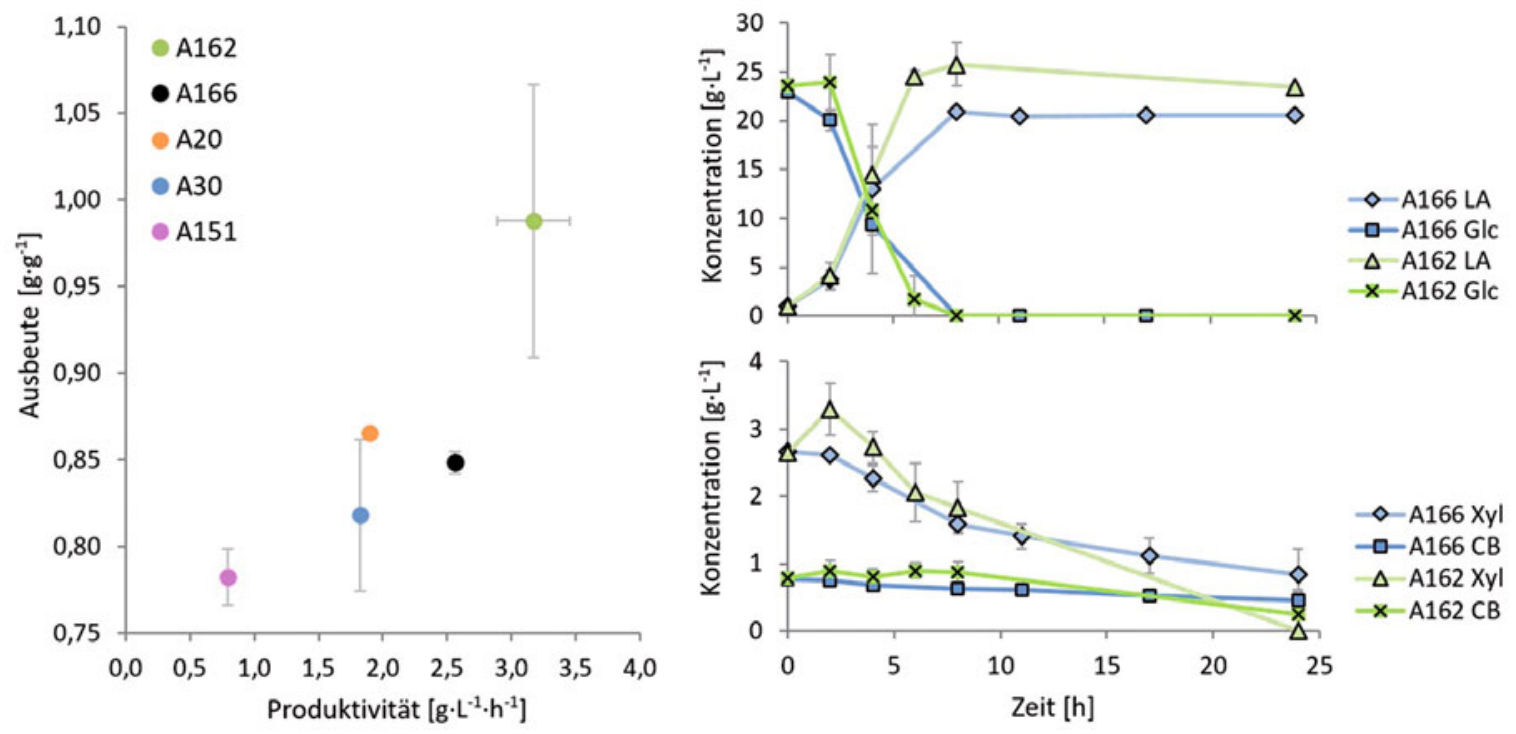

$\Delta$ Abb. 2: Ergebnisse des Stammscreenings (Bacillus coagulans, pH 5, $52{ }^{\circ} \mathrm{C}$ ); Mittelwerte mit Standardabweichung (Doppelbestimmung). LA: Milchsäure; Glc: Glucose; Xyl: Xylose; CB: Cellobiose. 

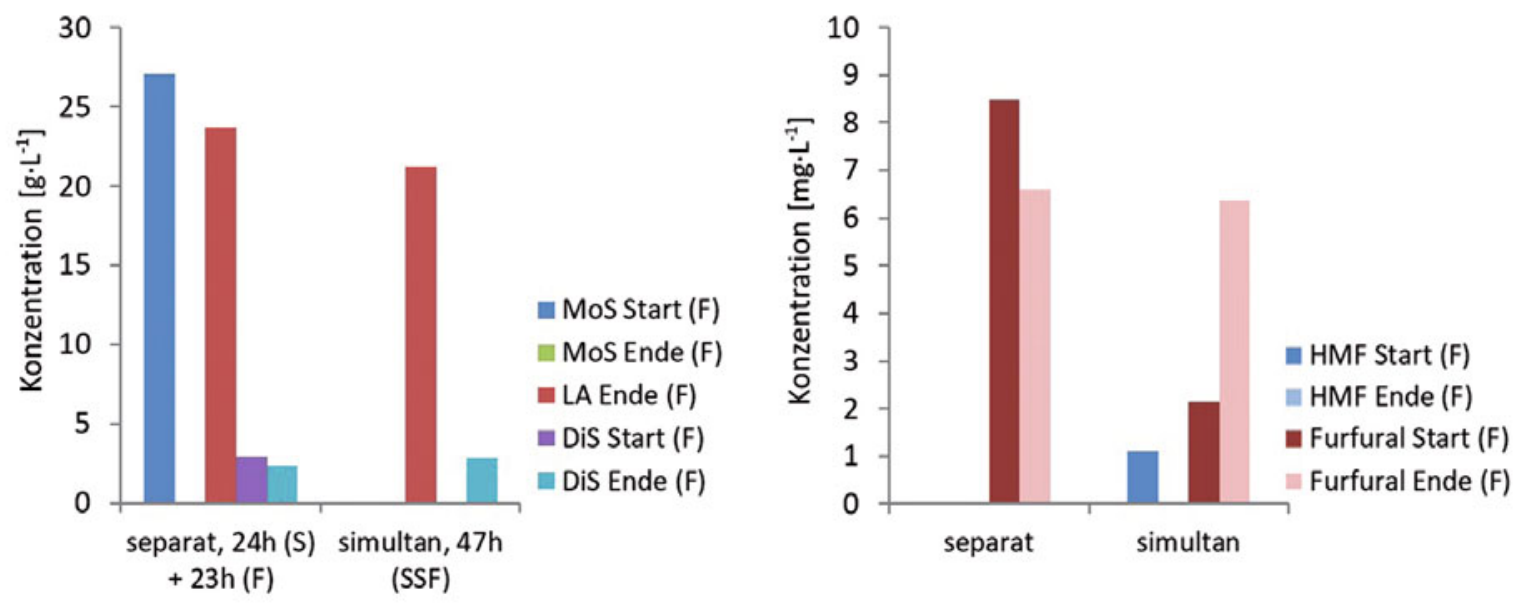

$\Delta$ Abb. 3: Vergleich von separater und simultaner Fermentation von LX-Cellulose durch Bacillus coagulans; links: Substrat- und Produktkonzentration, rechts: Inhibitorkonzentrationen. Start (F): Messung zu Beginn der Fermentation; Ende (F): Messung nach Abschluss der Fermentation. LA: Milchsäure; MoS: Monosaccharide; DiS: Disaccharide; HMF: Hydroxymethylfurfural.

innerhalb von 47 Stunden eine LA-Konzentration von $21,3 \mathrm{~g} \cdot \mathrm{L}^{-1}$ mit einer L-(+)-Reinheit von 99,4 Prozent erreicht (Abb. 3). Zusätzlich wurde für diese Versuche die Konzentration der Inhibitoren vor und nach der Fermentation gemessen: Furfural lag maximal mit $8,5 \mathrm{mg} \cdot \mathrm{L}^{-1}$ vor HMF mit $1,1 \mathrm{mg} \cdot \mathrm{L}^{-1}$. Diese Werte sind äußerst niedrig für aufgeschlossene Lignocellulose und können auf die milden Prozessbedingungen des LX-Verfahrens zurückgeführt werden. Das Wachstum von B. coagulans A166 wird aufgrund dieser geringen Konzentrationen sowie seiner Toleranz gegenüber Inhibitoren somit nicht beeinflusst.

Wie diese ersten Versuche zur Fermentation zeigen, kann eine SSF durchgeführt und der Prozess somit vereinfacht werden. Da im direkten Vergleich eine geringere Konzentration an LA im simultanen Versuch gewonnen wurde, werden im Projekt PoMiZu Versuche zur Optimierung des Prozesses verfolgt. Doch bereits jetzt führt das Projekt vor Augen, wie Biomasse kaskadisch genutzt werden kann: Aus dem landwirtschaftlichen Reststoff Stroh konnten Biogas, Lignin und im Anschluss polymerisierbare L-LA gewonnen werden. Die erfolgreiche Kombination des neuartigen LX-Verfahrens mit der Nutzung von B. coagulans ist somit ein zukunftsweisendes Beispiel für eine nachhaltige Wirtschaftsweise.

\section{Danksagung}

Die Autoren bedanken sich für die Förderung durch den Europäischen Fond für regionale Entwicklung der Europäischen Union mit freundlicher Unterstützung durch das Ministerium für Wirtschaft und Energie sowie der Investitionsbank des Landes Brandenburg (FKZ 80170179 und 80170137, ProFIT Brandenburg).

\section{Literatur}

[1] Koutinas AA, Vlysidis A, Pleissner D et al. (2014) Valorization of industrial waste and by-product streams via fermentation for the production of chemicals and biopolymers. Chem Soc Rev 43:2587-2627

[2] Laurichesse S, Avérous L (2014) Chemical modifications of lignins: towards biobased polymers. Prog Polym Sci 39: $1266-1290$

[3] Abdel-Rahman MA, Sonomoto K (2016) Opportunities to overcome the current limitations and challenges for efficient microbial production of optically pure lactic acid. J Biotechnol 236:176-192

[4] Cubas-Cano E, Venus J, González-Fernández C et al. (2020) Assessment of different Bacillus coagulans strains for 1-lactic acid production from defined media and gardening hydrolysates: effect of lignocellulosic inhibitors. J Biotechnol 323:9-16

[5] Aulitto M, Fusco S, Bartolucci S et al. (2017) Bacillus coagulans MA-13: a promising thermophilic and cellulolytic strain for the production of lactic acid from lignocellulosic hydrolysate. Biotechnol. Biofuels 10:210

[6] Schroedter L, Schneider R, Remus L et al. (2020) L-(+)Lactic Acid from Reed: Comparing Various Resources for the Nutrient Provision of B. coagulans. Resources 9:89

Funding note: Open Access funding enabled and organized by Projekt DEAL. Open Access: Dieser Artikel wird unter der Creative Commons Namensnennu 4.0 International Lizenzveröffentlicht, welche die Nutzung, Vervielfältigung. Bearbeitung, Verbreitung und Wiedergabe in jeglichem Medium und Forma erlaubt, sofern Sie den/die ursprünglichen Autor(en) und die Quelle ordnungsgemäß nennen, einen Link zur Creative Commons Lizenz beifügen un angeben, ob Änderungen vorgenommen wurden. Die in diesem Artikel enthaltenen Bilder und sonstiges Drittmaterial unterliegen ebenfalls der genannten Creative Commons Lizenz, sofern sich aus der Abbildungsle genannten Creative Com nach gesetzlichen Vorschriften erlaubt ist, ist für die oben aufgeführten Weiterverwendungen des Materials die Einwilligung des jeweiligen Rechteinhabers einzuholen. Weitere Details zur Lizenz entnehmen Sie bitte der Rechteinhabers einzuholen. Weitere Details zur Lizenz entnehmen Sie bitte der
Lizenzinformation auf http://creativecommons.org/licenses/by/4.0/deed.de.
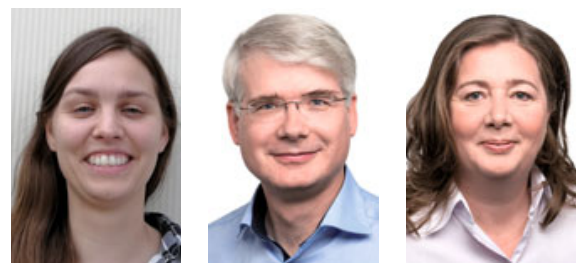

Linda Schroedter, Friedrich Streffer und Katrin Streffer (v.I.n.r.)
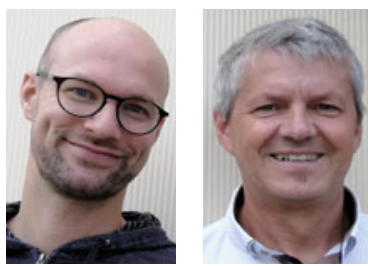

Peter Unger und Joachim Venus (rechts)

Korrespondenzadressen:

Dr. Joachim Venus

Leibniz-Institut für Agrartechnik und

Bioökonomie e. V.

Abteilung Bioverfahrenstechnik

Max-Eyth-Allee 100

D-14469 Potsdam

jvenus@atb-potsdam.de

www.atb-potsdam.de

Dr. Friedrich Streffer

Chief Technology Officer

LXP Group GmbH

Rheinstraße 3

D-14513 Teltow

friedrich.streffer@lxp-group.com

www.Ixp-group.com 